\title{
Slow light using photorefractive beam fanning
}

\author{
Nacera BOULDJA ${ }^{1,2, *}$, Marc SCIAMANNA ${ }^{1,2}$ and Delphine WOLFERSBERGER ${ }^{1,2}$
}

${ }^{1}$ Chair in Photonics, CentraleSupélec, LMOPS, F-57070 Metz, France

${ }^{2}$ Université Lorraine,CentraleSupélec,LMOPS, F-57070 Metz, France

*nacera.bouldja@centralesupelec.fr

Slow-down of the light velocity has potential applications in information processing [1] and interferometers. Recently, several works have experimentally [2] demonstrated the deceleration of light in photorefractive crystals using a two wave mixing (TWM). A low velocity value of the order of $0.02 \mathrm{~cm} / \mathrm{s}$ has been reported in $\mathrm{BaTiO}_{3}$ crystal for Gaussian pulse profiles of long duration of $1.3 \mathrm{~s}$ [3]. It was also reported that the time delay depends mainly on the coupling photorefractive gain. However, the photorefractive grating build-up time in that crystal does not allow to slow down pulses shorter than ms, which limits its interest for telecoms applications.

In this work, we use another property of photorefractive materials to decelerate light. We show the possibility of slowing-down a light pulse transmitted through a SPS crystal using the beam fanning effect. This asymmetric stimulated scattering is generated when a single incident beam propagates through a photorefractive crystal with a certain angle with respect to the c-axis [4]. In 2014, A. Grabar et al. [5] reported the speeding up of Gaussian pulses of durations in the range of $1 \mathrm{~ms}$ to $100 \mathrm{~s}$. Here we present an experimental result of slow light pulse of durations shorter than those studied in other photorefractive materials (like in $\mathrm{BaTiO}_{3}$ ). A delay of $113 \mu \mathrm{s}$ is observed for Gaussian pulse of full width duration at half- peak intensity of $600 \mu \mathrm{s}$, corresponding to a group velocity of $44 \mathrm{~m} / \mathrm{s}$.

Figure 1(a) shows the experiment setup . A laser beam of $638 \mathrm{~nm}$ is modulated by an electro-optic modulator (EOM) to be in a Gaussian shape. Then, it is transmitted through a $0.5 \mathrm{~cm}$-long SPS:Te crystal in z direction. The input and output pulses are recorded by two photodetectors $D_{1}$ and $D_{2}$.

(a)
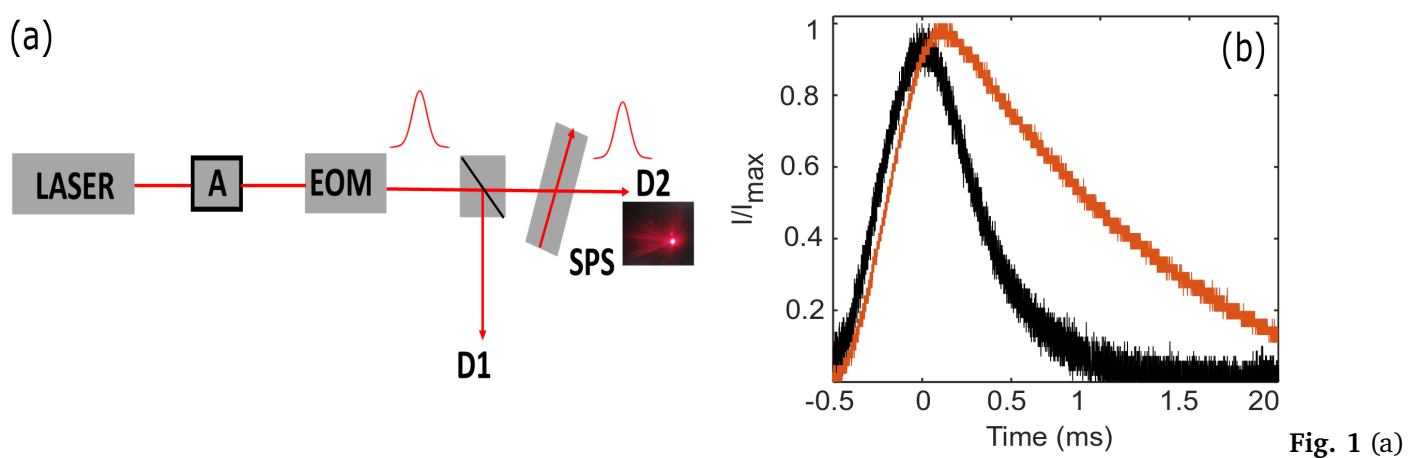

Experimental setup ; $E O M$ is the electrooptic modulator, $D_{i}$ are the detectors, $B S$ is the beamsplitters and A is the attenuator. (b) Temporal envelopes of the normalized input pulse (black line) and output pulse (red line) for input pulse duration of $t_{0}=600 \mu s$.

When the input pulse enters on the sample with an incidence angle $\theta=45^{\circ}$, the beam fanning appears in the direction of the transmitted pulse which is amplified, distorted and delayed at the output of the crystal. We show in Fig. 1(b) experimental measurements of the temporal envelope of the normalized input and output intensities as function of the time. The observed delay between the input and output intensities peaks is resulting from the photoreractive coupling of the input pulse with the beam fanning. If we change the incidence angle, the beam fanning intensity decreases and the pulse propagates in the crystal without any delay.

In conclusion, we have explained the origin of the delay of a single pulse in photorefractive crystal. We conclude that the beam fanning has a considerable effect on the group velocity of the pulse transmitted through the photorefractive crystal.

The Chaire Photonique is funded by: Ministry of Higher Education and Research, European Union (FEDER), Region Grand Est, Departement de la Moselle, Metz Metropole, Airbus-GDI Simulation, CentraleSupélec and Fondation Supélec. The authors also thank A.A. Grabar from the University of Uzhgorod for the SPS sample.

\section{References}


[1] Z. Dutton, and al, "Storing and processing optical information with ultraslow light in Bose-Einstein condensates," Phys. Rev. A.70, 053831 (2004).

[2] A. Shumelyuk and S. Odoulov, "Light pulse manipulation in $S n_{2} P_{2} S_{2}$,"J. Opt.12, 104015(2010).

[3] E. Podivilo and B. Sturman, "Light pulse slowing down up to $0.025 \mathrm{~cm} / \mathrm{s}$ by photorefractive two-wave coupling," Phys. Rev. Lett.91, 083902 (2003).

[4] Y. H. Hong, P. Xie, J. H. Dai, Y. Zhu, H. G. Yang, and H. J. Zhang, "Fanning effects in photorefractive crystals,"Opt Lett .18, 10 (1993).

[5] A. Grabar, P. Mathey, and G. Gadret, "Manipulation of fast light using photorefractive beam fanning,"J. Opt. Soc. Am. B, 31, 980 (0214). 